\title{
Prediction of Flow Fields around Ships by Modified Rankine Source Method
}

\author{
(1st Report)-Pilot Computations and Inviscid Case-
}

by Kazuhiro Mori*, Member Hitoshi Nishimoto**, Member

\begin{abstract}
Summary
A modified Rankine source method is proposed, where the free surface condition is linearized based on the viscous-double-hull flow and the hull surface condition is approximated to be satisfied only by the double-hull potential. The flow field is represented by Rankine sources distributed over the free surface.

Preliminary pilot computations for 2-D and 3-D point sources are carried out in the first place in order to establish the computation scheme. Then the availability of the present method is exemplified by computing the free surface flows and the wave elevations of M-21 and Wigley model whose hull forms are so simple that their double-hull flows can be prepared with least numerical errors. In this first report we confine ourselves to the inviscid case.

The higher approximation for the discrete source density is certified to be significant. The employment of the approximation for the hull-surface condition decreases both computing time and memory storages so much that the present method is promising for practical purposes. Free surface flows and wave elevations are well reproduced as far as the two models are concerned.
\end{abstract}

\section{Introduction}

The last workshop on ship-wave resistance computations $(1979)^{1)}$ shows clearly the present state of arts of wave resistance computations. The present research has originated from the assessment of its results ${ }^{2}$.

One of the difficulties in the free surface problems is the nonlinearity of the free surface condition. Though the exact nonlinear free surface condition is considered by direct numerical methods (Chan-Chan, etc.), the use of the doublehull linearized free surface condition seems to be most reasonable and promising for practical purposes. Dawson's results, which seem most winning among the workshop results, are those obtained by the Rankine source method with use of the double-hull-linearized free surface condition.

The Rankine source method where Rankine sources are distributed over the free surface was first tried by Gadd $(1976)^{3)}$. Dawson refined

* Faculty of Engineering, Hiroshima University ** West Japan Fluid Engineering Laboratory Co., LTD.
Gadd's intuitive way of solving into an elegant finite difference problem by applying Green's theorem. The method, however, requires so large memory storages and so long computing time that rather coarse mesh spacings are inevitably used. And such requirements of large memory storages and long computing time are pessimistic in practical applications.

In the discussion of ref. 2), it is pointed out that the violation of the hull surface condition by the free wave potential does not matter so seriously. The coincidence of Nakatake-Yamazaki's results with Dawson's may support this mention; they used the same free surface condition, but the former neglected the hull surface condition for the free wave potential while the latter made the total potential to satisfy it exactly. If this fact is approved, the computations get much simpler and much more finite mesh spacings for the free surface source can be employed, because the hull surface sources are not unknowns any more on the determination of the former source distribution.

The other difficulty is how to take viscous effects into account. Non than Mori included the viscous effects in their computations in the last 
workshop. Either important or not, they should be included in computations at least once in order to get a sound judgement on their neglection.

In the present paper, the viscous-double-hull free surface condition is derived and the free surface flows are calculated based on the Rankine source method together with examination of its numerical techniques. The inclusion of the viscous terms has been already tried in the previous paper ${ }^{4}$, but, because they are entrained onto the low speed theory, their effects were depicted only on the wave resistance. In the present scheme, however, more can be discussed such as free surface elevations and velocity fields.

The term of "modified" is added because the treatment of the hull and free surface conditions are different from that of Dawson's, as mentioned above. Computations of wave resistance and inclusion of viscous-double-hull flows are reserved for next report.

\section{Basic Equations}

\subsection{Expression of velocity potential by Rankine} source

We consider a ship fixed in a constant uniform flow of velocity $U$ in $x$-direction. $O-x y z$ is a referred Cartesian coordinate system whose origin is at the midship of undisturbed free surface; $z$-direction is upward.

The total velocity $\boldsymbol{q}$ is assumed to be of the decomposed form

$$
\boldsymbol{q}=\boldsymbol{i} U+\nabla \phi+\boldsymbol{q}_{\boldsymbol{V}},
$$

where $i$ : unit vector in $x$-direction, $\phi$ : perturbation velocity potential, $\boldsymbol{q}_{V}$ : viscous component in total velocity. And the continuity condition is assumed to be satisfied separately by

$$
\left.\begin{array}{l}
\nabla^{2} \phi=0, \\
\nabla \boldsymbol{q}_{V}=0 .
\end{array}\right\}
$$

Applying Green's theorem to $\phi$ and $1 / r$ in the fluid domain and its mirror, we get the integral expression of the velocity potential $\phi$ at $(x, y, z)$,

$$
\begin{aligned}
\phi(x, y, z)= & -\frac{1}{4 \pi} \iint_{S_{H}} \sigma_{H}\left(\frac{1}{r}+\frac{1}{r^{\prime}}\right) d S \\
& -\frac{1}{4 \pi} \iint_{S_{F}} \sigma_{F} \frac{1}{r} d S \\
& +\frac{1}{4 \pi} \iint_{\Sigma}\left\{\phi \frac{\partial}{\partial n}\left(\frac{1}{r}+\frac{1}{r^{\prime}}\right)\right. \\
& \left.-\frac{\partial \phi}{\partial n}\left(\frac{1}{r}+\frac{1}{r^{\prime}}\right)\right\} d S,
\end{aligned}
$$

where $r^{2}=\left(x-x^{\prime}\right)^{2}+\left(y-y^{\prime}\right)^{2}+\left(z-z^{\prime}\right)^{2}, \quad r^{\prime 2}=(x-$ $\left.x^{\prime}\right)^{2}+\left(y-y^{\prime}\right)^{2}+\left(z+z^{\prime}\right)^{2} ;\left(x^{\prime}, y^{\prime}, z^{\prime}\right)$ is a point on integral surface $S_{H}, S_{F}$ and $\Sigma$ which are hull surface, free surface and far vertical boundary planes including the computing domain respec- tively; $\sigma_{H}$ and $\sigma_{F}$ are source densities distributed on $S_{H}$ and $S_{F}$ respectively; $n$ is the normal into the fluid domain. In the reduction of Eq. (3), contributions from bottom and its image surface are not taken into account, which can be safely neglected, because of their symmetry, if the bottom is enough deep.

Now we split $\phi$ into three parts and equate them to each term of Eq. (3);

$$
\begin{aligned}
\phi= & \phi_{0}+\phi_{w}+\phi_{\infty}, \\
\phi_{0}= & -\frac{1}{4 \pi} \iint_{S_{H}} \sigma_{H}\left(\frac{1}{r}+\frac{1}{r^{\prime}}\right) d S, \\
\phi_{w}= & -\frac{1}{4 \pi} \iint_{S_{F}} \sigma_{F^{\prime}} \frac{1}{r} d S, \\
\phi_{\infty}= & \frac{1}{4 \pi} \iint_{\Sigma}\left\{\phi \frac{\partial}{\partial n}\left(\frac{1}{r}+\frac{1}{r^{\prime}}\right)\right. \\
& \left.-\frac{\partial \phi}{\partial n}\left(\frac{1}{r}+\frac{1}{r^{\prime}}\right)\right\} d S .
\end{aligned}
$$

$\phi_{0}$ is due to the hull surface source while $\phi_{w}$ is due to the free surface source. $\phi_{\infty}$ is the rest contribution from $\Sigma$ which may be truncated in terms of, e.g., the Havelock velocity potential. But it can be safely neglected if the computing domain is carefully chosen.

Here we introduce an approximation that $\phi_{0}$ is determined only from the hull surface condition;

$$
U \frac{\partial x}{\partial n}+\frac{\partial \phi_{0}}{\partial n}=0
$$

Then $\phi_{w}$ is determined in order for $\phi$ to satisfy the free surface condition.

This approximation is originally introduced by Baba $(1976)^{5)}$ in his low speed theory which allows for the hull surface condition not to be satisfied exactly; the free wave potential does not satisfy it. But according to the results of the last workshop, the violation of the hull surface condition by the free wave potential does not yield serious errors ${ }^{2}$. On the contrary, because unknowns on the hull disappear by this approximation, we can use so fine mesh spacings for the free surface unknowns that the computation can be carried out more accurately.

\subsection{Free surface condition}

Now we write the total velocity in another form

$$
\boldsymbol{q}=\nabla \phi_{w}+\boldsymbol{q}_{0}
$$

where $\boldsymbol{q}_{0}$ is the double-hull flow including viscous component; written by

$$
\boldsymbol{q}_{0}=\boldsymbol{i} U+\nabla \phi_{0}+\boldsymbol{q}_{V} .
$$

When the normal stress due to the viscosity is linearized, the free surface condition is given as follows ${ }^{6)}$.

Pressure condition; 


$$
g \zeta+\frac{1}{2}\left(\left|\nabla \phi_{w}+\boldsymbol{q}_{0}\right|^{2}-U^{2}\right)+g \delta H+2 \nu \frac{\partial w}{\partial z}=\left.0\right|_{z=\zeta,}
$$

where $g$ : gravity acceleration, $\zeta$ : free surface elevation, $w$ : $z$-direction component of $\boldsymbol{q}, \boldsymbol{v}$ : kinematic viscosity coefficient, and $\delta H$ is the head loss whose gradient is given in terms of vorticity $\omega$;

$$
g \nabla \delta H=\omega \times \boldsymbol{q}-\nu \nabla^{2} \boldsymbol{q} .
$$

The first term on RHS of Eq. (12) is the head loss due to the convection of vorticity and the second term is that due to the viscous diffusion.

Kinematic condition;

$$
\boldsymbol{q} \cdot \nabla(z-\zeta)=\left.0\right|_{z=\zeta}
$$

Eliminating $\zeta$ from Eqs. (11) (13), we get

$$
\left.\begin{array}{l}
\frac{1}{2}\left(\phi_{w x}+u_{0}\right)\left[\left(\phi_{w x}+u_{0}\right)^{2}+\left(\phi_{w y}+v_{0}\right)^{2}\right. \\
\left.\quad+\phi_{w z}^{2}+2 g \delta H\right]_{x} \\
+\frac{1}{2}\left(\phi_{w y}+v_{0}\right)\left[\left(\phi_{w x}+u_{0}\right)^{2}+\left(\phi_{w y}+v_{0}\right)^{2}\right. \\
\left.\quad+\phi_{w z}^{2}+2 g \delta H\right]_{y}+g \phi_{w z}=\left.0\right|_{z=0},
\end{array}\right\}
$$

where $u_{0}, v_{0}: x$ - and $y$-direction components of $\boldsymbol{q}_{0}$, and subscripts mean the differentiations with respect to the referred variables. In Eq. (14), the free surface conditions are approximately specified on $z=0$, and viscous terms are dropped.

If the nonlinear terms of $\phi_{w}$ are dropped and the relation for arbitrary function $F$

$$
u_{0} F_{x}+v_{0} F_{y}=q_{0} F_{l}
$$

is invoked, after Dawson $(1977)^{7}$, we get the free surface condition linearized by the viscous double-hull flow,

$$
\begin{aligned}
& q_{0}\left(q_{0} \phi_{w l}\right)_{l}+\phi_{w x}\left(q_{0} q_{0 x}+g \delta H_{x}\right)+\phi_{w y}\left(q_{0} q_{0 y}+g \delta H_{y}\right) \\
& \quad+q_{0} g \delta H_{l}+g \phi_{w z}=-\left.q_{0}{ }^{2} q_{0 l}\right|_{z=0},
\end{aligned}
$$

where $q_{0}:\left|\boldsymbol{q}_{0}\right|$, whose $x$-, $y$-components are $\boldsymbol{u}_{0}$, $v_{0} ; l$ : streamline along the viscous double-hull flow.

The head loss given by Eq. (12) is precisely discussed in Mori $(1979)^{6}$. If the viscous: diffusion term can be neglected, the gradients of head loss, appearing in Eq. (16), are written

$$
\left.\begin{array}{rl}
g \delta H_{x}= & -\omega_{z}\left(v_{0}+\phi_{w y}\right)+\omega_{y} \phi_{w z}, \\
g \delta H_{y}= & \omega_{z}\left(u_{0}+\phi_{w x}\right)-\omega_{x} \phi_{w z}, \\
g \delta H_{l}= & \frac{u_{0}}{q_{0}}\left(\omega_{y} \phi_{w z}-\omega_{z} \phi_{w y}\right) \\
& -\frac{v_{0}}{q_{0}}\left(\omega_{x} \phi_{w z}-\omega_{z} \phi_{w x}\right),
\end{array}\right\}
$$

where $\omega_{x}, \omega_{y}, \omega_{z}$ are components of $\omega$. Substituting Eq. (17) into Eq. (16), we get

$$
\begin{gathered}
q_{0}\left(q_{0} \phi_{w l}\right)_{l}+q_{0}\left(q_{0 x} \phi_{w x}+q_{0 y} \phi_{w y}\right)+g \phi_{w z} \\
+\phi_{w z}\left(u_{0} \omega_{\nu}-v_{0} \omega_{x}\right)=-\left.q_{0}{ }^{2} q_{0 l}\right|_{2=0} .
\end{gathered}
$$

Because the double-hull flow can not have nonzero value of $\omega_{x}$ and $\omega_{y}$ at $z=0$, they are supposed to be the vorticities induced by the free surface elevation. Then the last term on LHS of Eq. (18) may be a nonlinear term which can be dropped, though this term may play an important role when nonlinear effects are concerned. We get finally the viscous-double-hulllinearized free surface condition as follows;

$$
\begin{aligned}
& q_{0}\left(q_{0} \phi_{w l}\right)_{l}+q_{0}\left(q_{0 x} \phi_{w x}+q_{0 y} \phi_{w y}\right)+g \phi_{w z} \\
& \quad=-\left.q_{0}{ }^{2} q_{0 l}\right|_{2=0} .
\end{aligned}
$$

If the flow is inviscid, i.e., if the last term on RHS of Eq. (10) is zero, $q_{0 x}$ and $q_{0 y}$ get equal to $u_{0 l}$ and $v_{0 l}$ respectively. Then, by invoking Eq. (15) again, the first and the second terms on LHS of Eq. (19) can be reduced into one term and we get Dawson's double-hull-linearized free surface condition;

$$
\left(q_{0}^{2} \phi_{w l}\right)_{l}+g \phi_{w z}=-q_{0}^{2} q_{0 l}:
$$

Dawson's free surface condition of Eq. (20) still keeps the local curved flow effects while the free surface condition is simply imposed on the non-disturbed surface, $z=0$. (Discussions are made in Mori $(1980)^{2}$ on the double-hull linearized free surface conditions derived by Baba $\left.(1976)^{5}\right)$, Dawson $(1977)^{7}$ ) and YamazakiNakatake $(1979)^{8)}$ by relating each other.)

Comparing Eq. (19) with Eq. (20), no extra terms are appearing but the double-hull velocity $q_{0}$ includes the viscous component.

\subsection{Wave height, forces and moment}

Once the total velocity is determined, the free surface elevation $\zeta$ is given by Eq. (11) and the pressure is

$$
p=\frac{1}{2} \rho\left(U^{2}-|q|^{2}\right)-\rho g \delta H .
$$

Then the pressure resistance, $R_{p}$, the sinkage force, $T$ and the trim moment by stern, $M_{T}$ are given as follows;

$$
\begin{aligned}
& R_{p}=-\iint_{S_{H}} p n_{x} d S, \\
& T=\iint_{S_{H}} p n_{z} d S, \\
& M_{T}=\iint_{S_{H}} p n_{z}\left(x-x_{0}\right) d S .
\end{aligned}
$$

where $x_{0}: x$-coordinate of the center of gravity, $n_{x}, n_{y}: x-, y$-components of the outward normal on hull.

The above forces and moment will give new trim and sinkage conditions and computations may be iterated successively until the solution converges.

2.4 Determination of free surface source

The problem is now to determine the free sur- 
face source distribution $\sigma_{F}$ to satisfy Eq. (19).

We divide the free surface into M-pieces of small meshes whose areas are $\Delta S_{F j}$. Then quantities appearing in Eq. (19) are expressed by the sum notations;

$$
\begin{aligned}
\left.\phi_{w x}\right|_{z=0} & =\frac{1}{4 \pi} \sum_{j=1}^{M} \iint_{\Delta S_{F j}} \sigma_{F^{F}} \frac{x-x^{\prime}}{r_{0}^{3}} d x^{\prime} d y^{\prime} \\
& =\sum_{j=1}^{M} \sigma_{F j} U_{j}, \\
\left.\phi_{w y}\right|_{z=0} & =\frac{1}{4 \pi} \sum_{j=1}^{M} \iint_{\Delta S_{F j}} \sigma_{F} \frac{y-y^{\prime}}{r_{0}^{3}} d x^{\prime} d y^{\prime} \\
& =\sum_{j=1}^{M} \sigma_{F j} V_{j}, \\
\left.\phi_{w 2}\right|_{z=0} & =-\frac{1}{2} \sigma_{F i}, \\
\left.q_{0} \phi_{w i}\right|_{2=0} & =u_{0} \sum_{j=1}^{M} \sigma_{F j} U_{j}+v_{0} \sum_{j=1}^{M} \sigma_{F^{j}} V_{j},
\end{aligned}
$$

where $r_{0}{ }^{2}=\left(x-x^{\prime}\right)^{2}+\left(y-y^{\prime}\right)^{2}, \sigma_{F j}$ : source strength at the center of mesh, subscripts $i$ and $j$ mean the values at $\left(x_{i}, y_{i}\right)$ and $\left(x_{j}, y_{j}\right)$ respectively. Explicit expressions of $U_{j}, V_{j}$ are given in appendix.

Substituting Eqs. (25) (28) into Eq. (19), we get

$$
\begin{aligned}
& {\left[\sum_{j=1}^{M} \sigma_{F j} G_{i j}\right]_{l}+\sum_{j=1}^{M} \sigma_{F j} D_{i j}-\frac{1}{2} \frac{g}{q_{0}} \sigma_{F i}} \\
& \quad=-q_{0} q_{0 l},
\end{aligned}
$$

where

$$
\left.\begin{array}{l}
G_{i j}=u_{0 i} U_{j}+v_{0 i} V_{j}, \\
D_{i j}=q_{0 x i} U_{j}+q_{0 v i} V_{j} .
\end{array}\right\}
$$

Some appropriate expressions for the differentiation with respect to $l$ appearing in the first term on LHS of Eq.(29) will yield M-simultaneous equations for $\sigma_{F j}$.

\section{Pilot computations for 2-D and 3-D point sources}

In order to establish a numerical scheme, we carry out some pilot computations for 2-D and $3-D$ point sources where 1) finite difference forms, 2) upstream and downstream conditions and 3) mesh spacing, are examined. Such examinations are so important that the final results may depend on them; the dependency is not within so-called numerical errors and it is serious especially in wave problems.

The linearized free surface condition is

$$
\phi_{x x}+K_{0} \phi_{2}=\left.0\right|_{2=0},
$$

where $K_{0}=g / U^{2}$. The use of Eq. (31) instead of Eq. (19) does not loose generalities for the above purpose. Now consider 2-D and 3-D point sources at the origin of depth $-f$, whose strength is $\sigma_{0}$.
The velocity potentials due to them are given as follows;

2-D

$$
\begin{aligned}
\phi= & \frac{\sigma_{0}}{2 \pi}\left(\log \gamma_{2}+\log \gamma_{2}{ }^{\prime}\right) \\
& +\frac{1}{2 \pi} \int_{S_{F}} \sigma_{F} \log \left|x-x^{\prime}\right| d x^{\prime},
\end{aligned}
$$

3-D

$$
\phi=-\frac{\sigma_{0}}{4 \pi}\left(\frac{1}{r_{3}}+\frac{1}{r_{3}^{\prime}}\right)-\frac{1}{4 \pi} \iint_{S_{F}} \sigma_{F} \frac{1}{r_{0}} d x^{\prime} d y^{\prime},
$$

where $\quad r_{2}{ }^{2}=x^{2}+(z+f)^{2}, \quad r_{2}{ }^{2}=x^{2}+(z-f)^{2}, \quad r_{3}{ }^{2}=x^{2}$ $+y^{2}+(z+f)^{2}, r_{3}{ }^{\prime 2}=x^{2}+y^{2}+(z-f)^{2}$. The analytical expressions of wave elevation are given by $2-\mathrm{D}$

$$
\begin{aligned}
\zeta= & -\frac{U}{g} \frac{\sigma_{0}}{2 \pi}\left[\frac{2 x}{r_{2}^{2}}-2 \operatorname{sgn}(x)\right. \\
& \times \int_{0}^{\infty} m \frac{m \cos m f-K_{0} \sin m f}{m^{2}+K_{0}^{2}} e^{-m|x|} d m \\
& \left.-4 \pi K_{0} e^{-K_{0} f} \cos K_{0} x\right],
\end{aligned}
$$

3-D

$$
\begin{aligned}
\zeta= & -\frac{U}{g} \frac{\sigma_{0}}{4 \pi}\left[-\frac{2 K_{0}}{\pi} \int_{\theta-(\pi / 2)}^{\theta+(\pi / 2)} \sec \theta d \theta\right. \\
& \times \int_{0}^{\infty} m \frac{m \cos m f-K_{0} \sec ^{2} \theta \sin m f}{m^{2}+K_{0}^{2} \sec ^{4} \theta} e^{-m\lfloor\bar{\omega} \mid} d m \\
& -4 K_{0}^{2} \int_{\theta-(\pi / 2)}^{\pi / 2} \sec \theta e^{-K_{0} f \sec ^{2} \theta} \\
& \left.\times \cos \left(K_{0} \bar{\omega} \sec ^{2} \theta\right) d \theta\right]
\end{aligned}
$$

where $\bar{\omega}=x \cos \theta+y \sin \theta, \Theta=\tan ^{-1}(y / x)$. They are compared with numerical results.

Four-terms approximations for the first derivative of a function $F$ are given by

D-1:

$$
F^{\prime}=\frac{1}{6 h}\left(-2 F_{i-3}+9 F_{i-2}-18 F_{i-1}+11 F_{i}\right),
$$

D-2:

$$
F^{\prime}=\frac{1}{6 h}\left(-F_{i-3}+6 F_{i-2}-15 F_{i-1}+10 F_{i}\right) .
$$

In $\mathrm{D}-1$ form, higher terms than the third derivative in the Taylor expansion are neglected, while D-2 is a form which keeps up to the fourth derivative but drops the third. The latter is a form recommended in Dawson's original method ${ }^{7}$.

Usually the source strength is assumed constant within meshes. But this approximation sometimes brings forth significant numerical errors which can not be overlooked when tangen- 
tial velocities ( $\phi_{w x}$ and $\phi_{w y}$ in our problem) are concerned. This is because, if the source strength is constant within the $j$-th mesh, it induces no tangential velocity at $\left(x_{i}, y_{i}\right)$ when $i=j$. Here we assess the higher approximation proposed by Hess (1973) $)^{9)}$ comparing with the constant approximation. In the higher approximation, the source density on a mesh is expressed by a quadratic function of $s$,

$$
\sigma_{F}(s)=\sigma_{F j}+\sigma_{1} s+\sigma_{2} s^{2},
$$

where $s$ : the distance from the center of mesh, $\sigma_{1}$ and $\sigma_{2}$ are parameters determined by the use of neighboring mesh source strengthes of $\sigma_{F j-1}$ and $\sigma_{F j+1}$.

All the computations are carried out for $K_{0} f=$ 1.0 and $\sigma_{0}=2 \pi U / K_{0}$ and $4 \pi U / K_{0}^{2}$ for 2-D and 3-D respectively. Fig. 1 shows the dependency on finite difference forms, mesh spacings and the source strength approximations. The results obtained by the constant source approximation with the mesh spacing of $\Delta K_{0} x=0.25$ are short in wave length both for D-1 and D-2. Even an introduction of a smaller mesh spacing of $\Delta K_{0} x=$ 0.125 improves a little and the result is not still satisfying. To be worse, such a fine mesh spacing requires much greater memory storages which are never applicable to 3-D cases. The higher approximation for source strength, however, provides a appreciable improvement as seen in Fig. 1. D-2 form with the higher approximation may be recommendable. Though not shown here, neither more coarse mesh spacings nor other finite difference forms such as two- or threeterms approximations yielded better results.

Because Eq. (37) can not be applied to the upstream three points of $i=1,2$ and 3 , the two-points approximation.

$$
F^{\prime}=\frac{1}{h}\left(F_{i+1}-F_{i}\right)
$$

is employed. In Fig. 2 results obtained by the two different upstream conditions are compared;

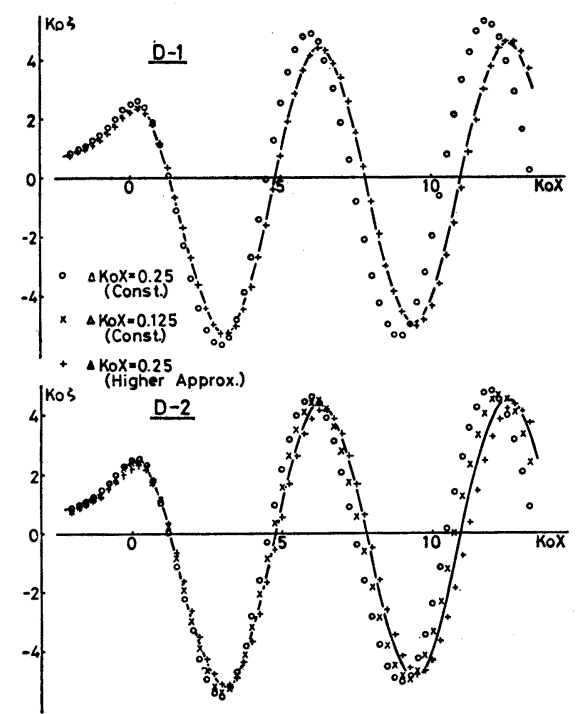

Fig. 1 Dependency on finite difference forms, mesh spacings and higher approximation for discrete source. (Computing domain: $-5.0 \leq K_{0} x \leq 20.0$ )

one is without any extra conditions and the other is under an imposition that no velocity is induced by the free surface sources at the upstream of $K_{0} x \leq-3.0$. Two computing domains of $-5.0 \leq$ $K_{0} x \leq 10.0$ and $-5.0 \leq K_{0} x \leq 20.0$ are also compared. The former differs much from the analytical result and the downstream reflection is appreciable. On the contrary, the latter closely coincides with the analytical result and the dependency on the computing domain is exactly non as far as results within $-5.0 \leq K_{0} x \leq 5.0$ are concerned.

Fig. 3 shows the dependency on the downstream reflection. If the results except within a half wave length from the terminating mesh of computation domain are referred, the downstream reflection is so humble that the computing
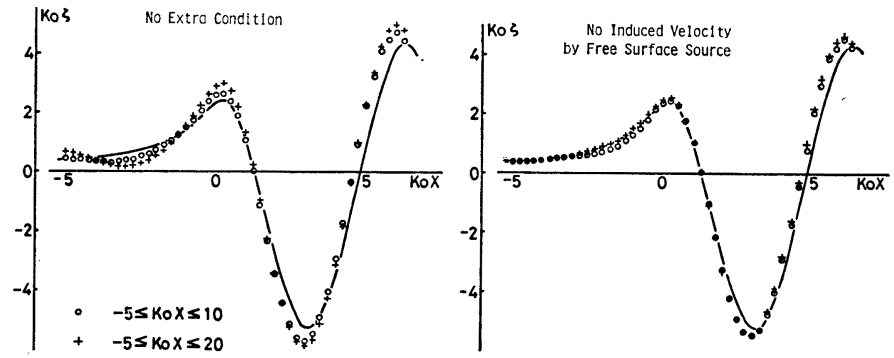

Fig. 2 Dependency on upstream condition (Mesh spacings: $\Delta K_{0} x=0.25 ; \mathrm{D}-2$ form with const. approx.) 


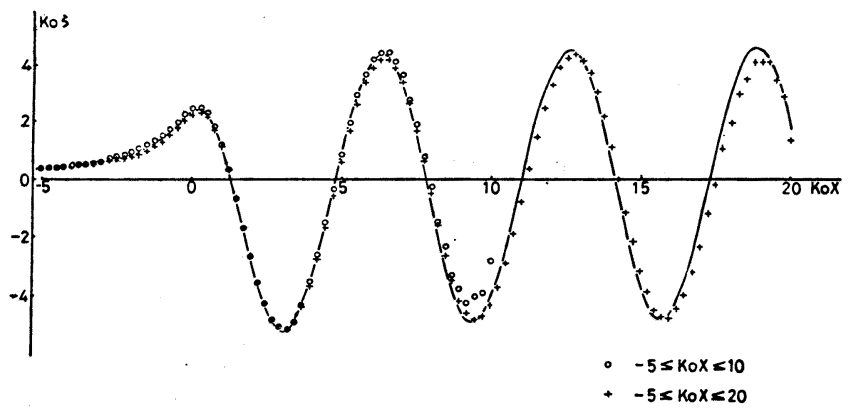

Fig. 3 Dependency on downstream reflection (Mesh spacings: $\Delta K_{0} x=0.25 ; \mathrm{D}-2$ form with higher approx.)

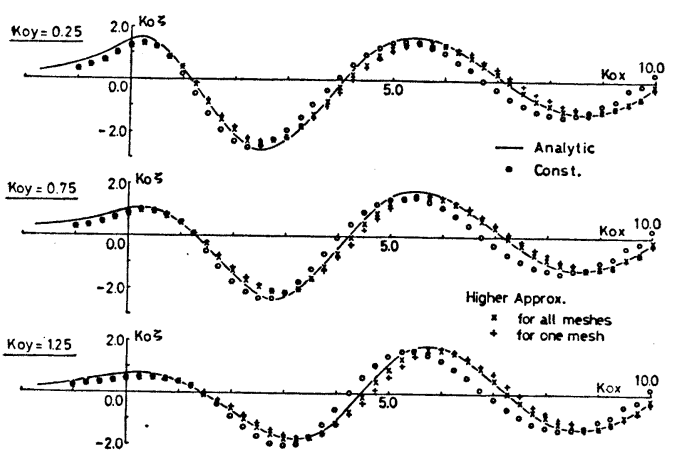

Fig. 4 Wave profiles of 3-D point source (Computing domain: $-3.0 \leq K_{0} x \leq$ $10.0,-4.0 \leq K_{0} y \leq 4.0$; mesh spacings: $\Delta K_{0} x=0.25, \Delta K_{0} y=0.5 ; \quad \mathrm{D}-2$ form)

domain which is wider than the reference region, at least, by one wave length may provide no serious errors.

Now we proceed to 3-D flow. Fig. 4 shows the wave profiles along three parallel cut lines of $K_{0} y=0.25,0.75$ and 1.25 ; the computing domain is $-3.0 \leq K_{0} x \leq 10.0$ and $-4.0 \leq K_{0} y \leq 4.0$, and the mesh spacing is $K_{0} x=0.25$ and $\Delta K_{0} y=0.5$. The results by the source constant approximation ( $O$ in Fig. 4) and by the higher approximation ( $x$ in Fig. 4) are compared with those calculated by Nakatake $(1966)^{10)}$ analytically. The higher approximation is taken into account only for $x$-direction here. The former shows poor agreements in this case too, while the latter is satisfying. In order to make the computing time less, we try to skip over the higher approximations for all the meshes except the very same mesh on which the velocity is computed. The results ( + in Fig. 4) do not infect seriously. But it serves much in reducing computing time. This approximation is used throughout the following

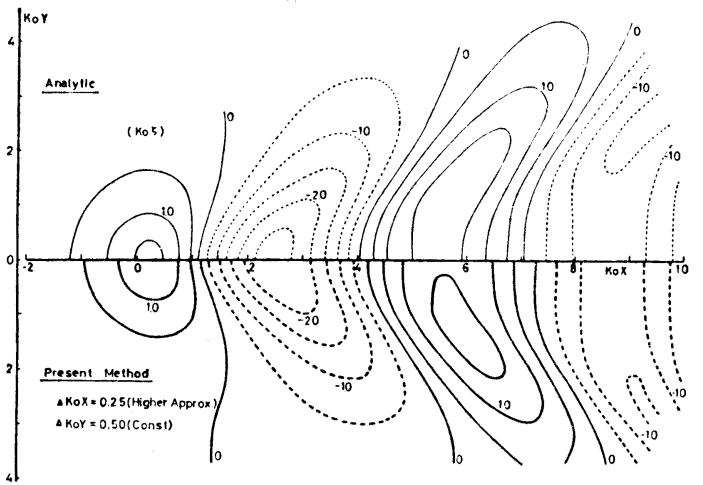

Fig. 5 Wave patterns of 3-D point source (D-2 form with higher approx.; other computing conditions are same as Fig. 4)

\section{3-D computations.}

In Fig. 5 the wave pattern obtained by the present method is compared with the analytical one. Though slight discrepancies are observed in the region close to the center line $(y=0)$, they agree rather well with each other, which guarantees us to apply the present method to 3-D ship wave problems.

\section{Computations for simple hull forms}

In order to exemplify the availability of the present method, computations for simple hull forms are carried out; Inuid M-21 and Wigley model. The former use is to be free from numerical errors in the double-hull flow calculations. (In the present report we confine ourselves to inviscid cases.)

The source distribution of M-21 is given by

$$
\sigma_{0} / U=a_{1} \xi+a_{3} \xi^{3}+a_{5} \xi^{5} \quad(\xi=2 x / L)
$$

on its centerplane of $2 z / L \geq-0.08$, where $a_{1}=$ $2.17444, a_{3}=-4.11286$ and $a_{5}=2.25910 ; L$ : 
ship length. Experimental data are referred from Mori et al (1972) ${ }^{11}$ and Kitazawa et al $(1974)^{12)}$

Fig. 6 is the mesh arrangement used in the present computations. The computation domain is as wide as $-1.5 \leq 2 x / L \leq 3.0$ and $-0.9 \leq 2 y / L$ $\leq 0.9$. Meshes are not equally spaced but more finitely around the centerplane, bow and stern. The total number of meshes is 477 for $y \geq 0$. Results are shown in Figs. $7 \sim 11$.

Fig. 7 shows the free surface source distributions along mesh rows, where $m$ is the mesh row number shown in Fig. 6. Fig. 8 is the comparison of perturbation velocity along a line where measurements are carried out. The line is shown also in Fig. 6. The measurements are done just beneath the free surface departing by $2 z / L=0.02$, while computations are on $z=0$. As a whole, it is rather well reproduced by computations. But a careful comparison will show that $u$-component is less in the vicinity of bow, which brings forth less wave height as discussed later. The neglection of the free surface slope might be one of the reason for this departure, which can be taken into account in the second iteration, if necessary. It is also observed that $v$-component is undesirably undulating around $2 x / L=-0.5$. Such a tendency is observed in

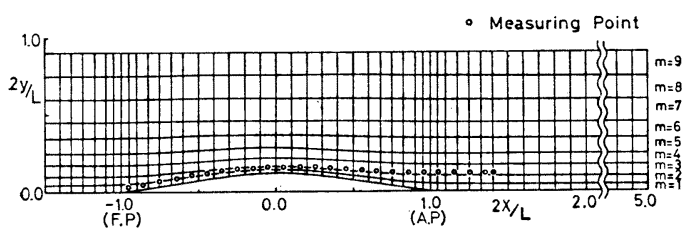

Fig. 6 Mesh system for M-21 and measuring points

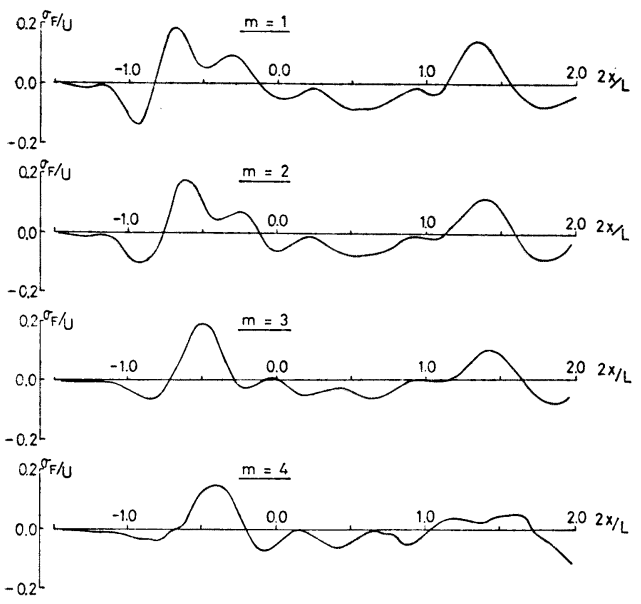

Fig. 7 Free-surface-source distributions of M-21 $(F n=0.289)$ $w$-component also but with a slight phase shift.

In Fig. 9, free surface elevations are compared along the same line as the velocity comparison. There are shown two measured wave heights; one is directly measured, and the other is the estimated one from Eq. (11) by the substitution of the measured velocity. Though it is out of our present scope, there can be observed a significant discrepancy between the above two "measured" waves in the first peak, which may throw a doubt on the use of Eq. (11) at $z=0$. Returning to the computed results, they show good agreement with measured except near the bow. The computed result is still appreciably less compared even with the latter "measured" wave height. This is mainly because of the underestimation in $u$-component as mentioned above. The no-free-wave condition at the upstream is well realized. On the other hand, there observed an unrealistic flucturation in the very vicinity of bow. This may be attributed to the violation of the hull surface condition by $\phi_{w}$. This is because, as seen later, such fluctuations are not observed in case of a Wigley model which is much more slender.

Fig. 10 shows the comparison of wave patterns

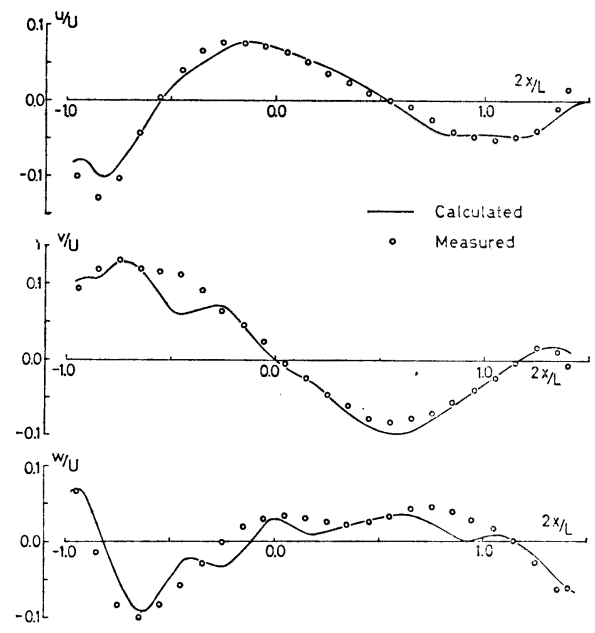

Fig. 8 Comparisons of velocity components of M-21 $(F n=0.289)$

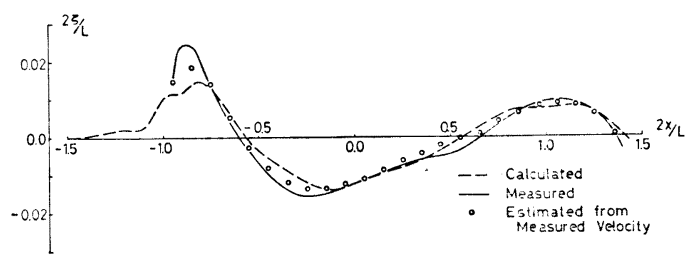

Fig. 9 Comparison of free surface elevation of M-21 $(F n=0.289)$ 


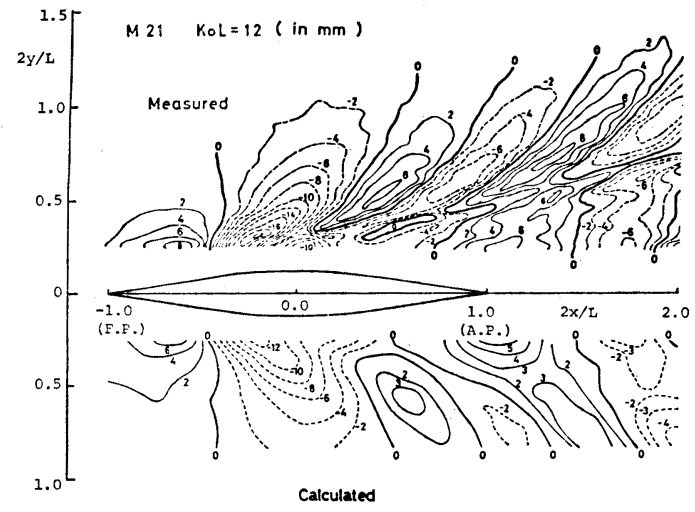

Fig. 10 Comparison of wave contour of $\mathrm{M}-21(F n=0.289)$

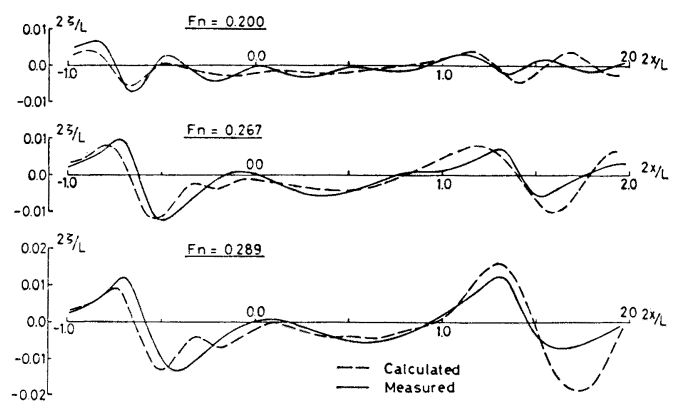

Fig. 11 Comparison of wave profiles of Wigley model at $2 y / L=0.13(F n=$ $0.200,0.267$ and 0.289 )

at $F_{n}=0.289$. As a whole computations are well reproducing but slight phase shifts are observed. And wave elevations are less than measured, especially in stern waves. This is because M-21 is an optimized hull and wave generations are oppressed more effectively in inviscid theory. So the inclusion of viscous terms may partially contrive this discrepancy. (It is worthy to notice that in case of M-21 the theoretical wave resistance is less than that of experiment.)

Next we carry out computations for a Wigley model of $B / L=0.1$. In Fig. 11, comparisons are made in parallel-cut wave profiles, at $2 y / L=$ 0.13 , at three speeds of $F_{n}=0.20,0.267$ and 0.289 . The double-hull flow is calculated numerically by dividing the hull surface into 120 panels. The free surface mesh arrangement is exactly the same as that of M-21 in $x$-direction but only six mesh rows are adopted. There are slight discrepancies between computed and measured which are a little more noticeable than those observed in M-21. They are partially attributed to the narrower computing domain and partially to the accuracy of double-hull flows. The result of $F_{n}=0.200$ shows poorer agreements than others; this is because the mesh spacing decreases relatively as the velocity decreases. The neglection of viscosity may be also responsible for the discrepancies observed in stern wave regions, which will be discussed in the 2 nd report.

Though there are several rooms for numerical improvements, we can conclude from the above two examples that the present modified Rankine source method is promising to estimate flow fields around practical ships with free surfaces. The computing time and memory storages are so modest that computations can be carried out as routine works; in case of M-21, the computing time is less than ten minutes by the M-200 HITAC machine when the double-hull flows are provided.

\section{Conclusions}

Following remarks can be mentioned as conclusions of the present paper.

1) A modified Rankine source method is proposed which is based on the viscous-doublehull-linearized free surface condition. It will serve for future discussions on the viscous effects on the wave resistance and the free surface flows.

2) The employed approximation that the hull surface condition is satisfied only by the doublehull potential provides savings of memory storages and computing time and it affords to use more finite free surface mesh spacings which have yielded accurate results.

3) The upstream condition that no velocity is induced by the free surface sources yields a reasonable result.

4) Except the region within a single or a half wave length from the terminating meshes of the computation domain, no significant downstream reflections are appreciable.

5) Higher approximations for the free surface source whose tangential velocity components play an important role provide significant improvement for the final results.

6) As far as the free surface flows and the free surface elevations of simple hull forms of M-21 and a Wigley model are concerned, the present method reproduces quite promising results for practical applications.

Authors wish to express their gratitude to Professor S. Hatano of Hiroshima University for his discussions through the present study. They also express their appreciation to Mr. K. Murata, a post graduate student of Hiroshima University, for his assistant in computations.

All computations are carried out by HITAC M-180 and M-200 of Hiroshima University Information Processing Center. 


\section{References}

1) Proceedings of the Workshop on ShipWave Resistance Computations, Edited by K. J. Bai and J. H. McCarthy (1979).

2) Mori, K.: Comments on Papers Presented at the Workshop on Wave Resistance, JTTC Report No. 1980-I-1 (1980).

3) Gadd, G. E.: A Method of Computing the Flow and Surface Wave Pattern around Full Forms, Trans. of RINA, Vol. 118 (1976).

4) Mori, K.: Calculation of Near Wake Flow and Resistance of Elliptic-Waterplane Ships, Proc. 13th Symposium on Naval Hydrodynamics (1981).

5) Baba, E.: Wave Resistance of Ships in Low Speed, MTB Vol. 109 (1976).

6) Mori, K.: Prediction of Viscous Effects on Wave Resistance of Ship in Framework of Low Speed Wave Resistance Theory, Mem. of Faculty of Eng. Hiroshima Univ., Vol. 7, No. 1 (1979).

7) Dawson, C. W.: A Practical Computer Method for Solving Ship-Wave Problems, Proc. of 2nd Intern. Conf. on Numerical Ship Hydrodynamics (1977).

8) Yamazaki, R., Nakatake, K., Nakamura, M.: Low Speed Wave-Resistance Theory Making Use of Strained Coordinates, IJR Research Report 1978 (1979).

9) Hess, J. L.: Higher Order Numerical Solution of the Two-Dimensional Neumann Problem, Computer Methods in Applied Mechanics and Engineering, Vol. 2 (1973).

10) Nakatake, K.: On the Wave Pattern Created by Singular Points, Trans. of West-Japan Soc. of Nav. Arch., Vol. 31 (1966), (in Japanese).

11) Mori, K., Inui, T., Kajitani, H.: Analysis of Ship-Side Wave Profiles with Special Reference to Hull's Sheltering Effect, Proc. of 9th Symposium on Naval Hydrodynamics (1972).

12) Kitazawa, T., Inui, T., Kajitani, H.: Velocity Field Measurements Applied for Analysis of Ship's Wave-Making Singularities, Proc. of 10th Symposium on Naval Hydrodynamics (1974).

\section{Appendix}

Here explicit expressions for $U_{j}$ and $V_{j}$ of Eqs. (25) and (26) are given. As in the text, $u$ and $v$ at $\left(x_{i}, y_{i}\right)$ are given by

$$
\left.\begin{array}{l}
u=\frac{1}{4 \pi} \sum_{j=1}^{M} \iint_{\Delta S_{F j}} \sigma_{F} \frac{x-x^{\prime}}{r_{0}^{3}} d x^{\prime} d y^{\prime} \\
v=\frac{1}{4 \pi} \sum_{j=1}^{M} \iint_{\Delta S_{F j}} \sigma_{F} \frac{y-y^{\prime}}{r_{0}{ }^{3}} d x^{\prime} d y^{\prime} .
\end{array}\right\}
$$

$\Delta S_{F j}$ is a subdivided mesh whose ordinates are always parallel to $y$-coordinate, as shown in Fig. A1. We introduce new variables $s^{\prime}$ and $t^{\prime}$ defined by

$$
\left.\begin{array}{l}
s^{\prime}=x^{\prime}-x_{i}, \\
t^{\prime}=y^{\prime}-y_{i} .
\end{array}\right\}
$$

Then the higher approximation for source strength $\sigma_{F}$, given by Eq. (38) in the text, is written

$$
\begin{aligned}
\sigma_{F}= & \sigma_{0}+\sigma_{1} X_{i j}+\sigma_{2} X_{i j}^{2} \\
& +\left(\sigma_{1}+2 \sigma_{2} X_{i j}\right) s^{\prime}+\sigma_{2} s^{\prime 2},
\end{aligned}
$$

when it is adopted only for $x$-direction; where $X_{i j}=x_{i}-x_{j}$.

We employ the higher approximation of Eq. (A3) for $u$-component while the constant approximation for $v$-component. Then Eq. (A1) is

$$
\begin{aligned}
u= & -\frac{1}{4 \pi} \sum_{j=1}^{M} \int_{s_{1}}^{s_{2}} s^{\prime} d s^{\prime} \int_{t_{1}}^{t_{2}} \\
& \times \frac{\sigma_{0}+\sigma_{1} X_{i j}+\sigma_{2} X_{i j}^{2}+\left(\sigma_{1}+2 \sigma_{2} X_{i j}\right) s^{\prime}+\sigma_{2} s^{\prime 2}}{\left(s^{\prime 2}+t^{\prime 2}\right)^{3 / 2}} \\
& \times d t^{\prime}, \\
v= & \left.-\frac{1}{4 \pi} \sum_{j=1}^{M} \sigma_{F j} \int_{s_{1}}^{s_{2}} d s^{\prime} \int_{t_{1}}^{t_{2}} \frac{t^{\prime}}{\left(s^{\prime 2}+t^{\prime 2}\right)^{3 / 2}} d t^{\prime}, \text { (A } 5\right)
\end{aligned}
$$

where

$$
\begin{aligned}
& s_{m}=x_{i}-\bar{x}_{m}, \quad t_{n}=a_{n} s^{\prime}+b_{n}, \quad(m, n=1,2) \\
& a_{1}=\frac{\bar{y}_{3}-\bar{y}_{1}}{\bar{x}_{2}-\bar{x}_{1}}, \quad a_{2}=\frac{\bar{y}_{4}-\bar{y}_{2}}{\bar{x}_{2}-\bar{x}_{1}}, \\
& b_{1}=\bar{y}_{1}-y_{i}-a_{1}\left(\bar{x}_{1}-x_{i}\right), \\
& b_{2}=\bar{y}_{2}-y_{i}-a_{2}\left(\bar{x}_{1}-x_{i}\right),
\end{aligned}
$$

and $\bar{x}_{m}$ and $\bar{y}_{m}$ are coordinates of mesh corners, as shown in Fig. A1.

After integration, Eq. (A4) is

$$
u=-\frac{1}{4 \pi} \sum_{j=1}^{M}\left(S_{22}-S_{21}-S_{12}+S_{11}\right) \text {, }
$$

where

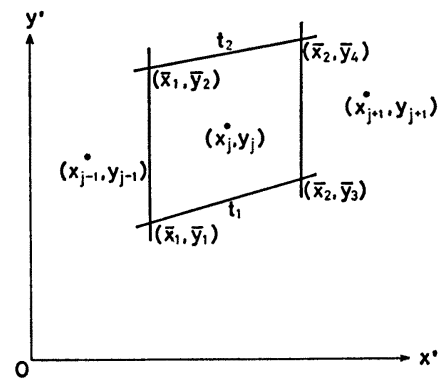

Fig. A1 Definition sketch 
$S_{m n}=\sigma_{0} A_{m n}+\sigma_{1} B_{m n}+\sigma_{2} C_{m n}$, and

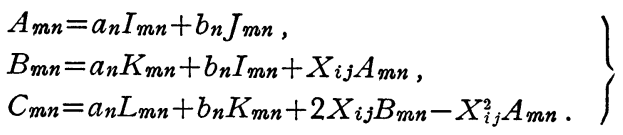

$I_{m n}, J_{m n}, K_{m n}$ and $L_{m n}$ are given as follows;

$$
\begin{aligned}
I_{m n}= & \frac{1}{\sqrt{1+a_{n}^{2}}} \log \mid\left(1+a_{m}^{2}\right) s_{m}+a_{n} b_{n} \\
& +\sqrt{\left(1+a_{n}^{2}\right) R_{m n}} \mid \\
J_{m n}= & \frac{1}{\left|b_{n}\right|} \log \left|\frac{\sqrt{1+a_{n}^{2}} s_{m}+\left|b_{n}\right|-\sqrt{R_{m n}}}{\sqrt{1+a_{n}^{2}} s_{m}-\left|b_{n}\right|-\sqrt{R_{m n}}}\right| \\
K_{m n}= & \frac{1}{1+a_{n}^{2}}\left(\sqrt{R_{m n}}-a_{n} b_{n} I_{m n}\right) \\
L_{m n}= & \frac{1}{2\left(1+a_{n}^{2}\right)}\left[\left\{\left(1+a_{n}^{2}\right) s_{m}-3 a_{n} b_{n}\right\} \sqrt{R_{m n}}\right. \\
& \left.+\left(2 a_{n}^{2}-1\right) b_{n}^{2} I_{m n}\right]
\end{aligned}
$$

and

$$
R_{m n}=\left(1+a_{n}^{2}\right) s^{2}+2 a_{n} b_{n} s_{m}+b_{n}^{2},
$$

On the other hand, $\sigma_{0}, \sigma_{1}$ and $\sigma_{2}$ are given as follows by the use of the neighboring source strength of $\sigma_{F j-1}$ and $\sigma_{F^{j+1}+1}$.

$$
\begin{aligned}
\sigma_{0}= & \sigma_{F}, \\
\sigma_{1}= & -\frac{x_{j+1}-x_{j}}{\left(x_{j}-x_{j-1}\right)\left(x_{j+1}-x_{j-1}\right)} \sigma_{F j-1} \\
& +\frac{x_{j+1}-2 x_{j}+x_{j-1}}{\left(x_{j}-x_{j-1}\right)\left(x_{j+1}-x_{j}\right)} \sigma_{F j} \\
& +\frac{x_{j}-x_{j-1}}{\left(x_{j+1}-x_{j}\right)\left(x_{j+1}-x_{j-1}\right)} \sigma_{F j+1} \\
\sigma_{2}= & \frac{1}{\left(x_{j}-x_{j-1}\right)\left(x_{j+1}-x_{j-1}\right)} \sigma_{F j-1} \\
& -\frac{1}{\left(x_{j}-x_{j-1}\right)\left(x_{j+1}-x_{j}\right)} \sigma_{F j} \\
& +\frac{1}{\left(x_{j+1}-x_{j}\right)\left(x_{j+1}-x_{j-1}\right)} \sigma_{F j+1}
\end{aligned}
$$

Substituting them into Eq. (A8), we get

$$
\begin{aligned}
S_{m n}= & -\frac{\left(x_{j+1}-x_{j}\right) B_{m n}-C_{m n}}{\left(x_{j}-x_{j-1}\right)\left(x_{j+1}-x_{j-1}\right)} \sigma_{F j-1} \\
& +\left\{A_{m n}+\frac{\left(x_{j+1}-2 x_{j}+x_{j-1}\right) B_{m n}-C_{m n}}{\left(x_{j+1}-x_{j}\right)\left(x_{j}-x_{j-1}\right)}\right\} \sigma_{F j} \\
& +\frac{\left(x_{j}-x_{j-1}\right) B_{m n}+C_{m n}}{\left(x_{j+1}-x_{j}\right)\left(x_{j+1}-x_{j-1}\right)} \sigma_{F j+1}
\end{aligned}
$$

Then $u$ is given by

$$
\begin{aligned}
\varkappa= & \frac{1}{4 \pi} \sum_{j=1}^{M}\left[-\frac{\left(x_{j+1}-x_{j}\right) B_{j}-C_{j}}{\left(x_{j}-x_{j-1}\right)\left(x_{j+1}-x_{j-1}\right)} \sigma_{F j-1}\right. \\
& +\left\{A_{j}+\frac{\left(x_{j+1}-2 x_{j}+x_{j+1}\right) B_{j}-C_{j}}{\left(x_{j+1}-x_{j}\right)\left(x_{j}-x_{j-1}\right)} \sigma_{F j}\right. \\
& \left.+\frac{\left(x_{j}-x_{j-1}\right) B_{j}+C_{j}}{\left(x_{j+1}-x_{j}\right)\left(x_{j+1}-x_{j-1}\right)} \sigma_{F j+1}\right]
\end{aligned}
$$

where

$$
\left.\begin{array}{l}
A_{j}=A_{22}-A_{21}-A_{12}+A_{11}, \\
B_{j}=B_{22}-B_{21}-B_{12}+B_{11}, \\
C_{j}=C_{22}-C_{21}-C_{12}+C_{11} .
\end{array}\right\}
$$

Now, if the higher approximation is applied only for the mesh of $j=i$, then $U_{j}$ of Eq. (25) in the text is given as follows;

$$
\begin{aligned}
& U_{j}=\frac{1}{4 \pi}\left[A_{j}-\frac{\left(x_{j_{+2}}-x_{j+1}\right) B_{j+1}-C_{j_{+1}}}{\left(x_{j+1}-x_{j}\right)\left(x_{j+2}-x_{j}\right)}\right], \\
& \text { for } j=i-1 \\
& U_{j}=\frac{1}{4 \pi}\left[A_{j}+\frac{\left(x_{j+1}-2 x_{j}+x_{j-1}\right) B_{j}-C_{j}}{\left(x_{j+1}-x_{j}\right)\left(x_{j}-x_{j-1}\right)}\right] \text {, } \\
& \text { for } j=i \\
& U_{j}=\frac{1}{4 \pi}\left[A_{j}+\frac{\left(x_{j-1}-x_{j-2}\right) B_{j-1}+C_{j-1}}{\left(x_{j}-x_{j-1}\right)\left(x_{j}-x_{j-2}\right)}\right] \text {, } \\
& \text { for } j=i+1 \\
& U_{j}=\frac{1}{4 \pi} A_{j} . \\
& \text { for } j \neq i-1, i, i+1
\end{aligned}
$$

All $X_{i j}$ 's included in Eq. (A16) are zero.

For the terminating meshes, we treat as follows. For the upstream termination, $\sigma_{F j-1}$ is assumed to be zero. And for the downstream, a linear approximation is adopted, i.e., $\sigma_{2}$ is dropped from Eq. (A3). Then we get

$$
S_{m n}=\left(A_{m n}+\frac{C_{m n}}{x_{j}-x_{j-1}}\right) \sigma_{F j}-\frac{B_{m n}}{x_{j}-x_{j-1}} \sigma_{F j-1} .
$$

Eq. (A5) is easily integrated and $V_{j}$ is given by

$$
V_{j}=I_{22}-I_{21}-I_{12}+I_{11}
$$

where $I_{m n}$ is given by Eq. (A10). 